\title{
Role of immunotherapy in Bacillus Calmette-Guérin unresponsive: non-muscle invasive bladder cancer
}

\author{
Jungyo Suh, Sangjun Yoo \\ Department of Urology, Seoul National University Boramae Medical Center, Seoul, Korea \\ Contributions: (I) Conception and design: S Yoo; (II) Administrative support: S Yoo; (III) Provision of study materials or patients: S Yoo; (IV) \\ Collection and assembly of data: J Suh; (V) Data analysis and interpretation: J Suh; (VI) Manuscript writing: All authors; (VII) Final approval of \\ manuscript: All authors. \\ Correspondence to: Sangjun Yoo, MD, PhD. Department of Urology, Seoul National University Boramae Medical Center, 5 Gil 20, Boramae-Road, \\ Dongjak-Gu, Seoul 07061, Korea. Email: ebend@naver.com.
}

\begin{abstract}
Bacillus Calmette-Guérin (BCG) is recommended as the first-line treatment option for intermediate to high risk non-muscle invasive bladder cancer (NMIBC) by current clinical guidelines. However, despite the intravesical instillation of BCG, a significant proportion of patients with intermediate to high risk NMIBC develop intravesical recurrence. Moreover, the treatment of BCG-unresponsive NMIBC is currently challenging. There are no reliable treatment options for these patients with BCGunresponsive NMIBC except radical cystectomy, which reported to show acceptable oncological outcomes. In this regards, reliable and safe non-invasive or less-invasive treatment options with acceptable oncological outcomes are awaited for the treatment of BCG-unresponsive NMIBC. The treatment of advanced or metastatic urothelial carcinoma has greatly advanced following the recent introduction of immunotherapeutic agents. These advancements have triggered an increasing interest in the use of immunotherapeutic agents for NMIBC, and especially for BCG-unresponsive NMIBC. The current review article aims to introduce and discuss the cutting-edge knowledge on the role of immunotherapy in BCG-unresponsive NMIBC and the currently available therapeutic strategies for its treatment. In addition, this article also summarizes the ongoing studies in this field.
\end{abstract}

Keywords: Administration; intravesical; Bacillus Calmette-Guérin vaccine (BCG vaccine); immunotherapy; urinary bladder neoplasms

Submitted Jan 29, 2020. Accepted for publication Apr 29, 2020.

doi: $10.21037 /$ tcr-20-758

View this article at: http://dx.doi.org/10.21037/tcr-20-758

\section{Introduction}

Non-muscle invasive bladder cancer (NMIBC) is confined to the mucosa and submucosa, and comprises approximately $75 \%$ of initially diagnosed bladder cancers (1). Although most of the patients with NMIBC are easily treated by the transurethral resection of bladder tumors, up to $80 \%$ and $15 \%$ of patients experience recurrence and progression, respectively, within five years $(2,3)$. There are several therapeutic options for reducing the recurrence and progression of NMIBC. Among these, the intravesical instillation of Bacillus Calmette-Guérin (BCG) immunotherapy is more effective in reducing the rate of recurrence than the intravesical instillation of most of the prescribed chemotherapeutic agents (4).

The intravesical instillation of BCG is recommended as the standard treatment for intermediate to high risk NMIBC by current clinical guidelines $(5,6)$. However, despite the intravesical instillation of BCG, a significant proportion of patients with NMIBC develop recurrence, and effective treatment options for BCG unresponsive NBIMC are limited. Radical cystectomy is the preferred treatment option in case of BCG treatment failure, however, bladder preservation or repeated intravesical treatments 
can be considered in certain situations (6). Owing to the high mortality and morbidity of radical cystectomy, it is necessary to identify alternatives for the clinical treatment of patients with NMIBC who have failed on BCG therapy.

Recent advancements in the field of immuneoncological drugs have demonstrated their promising effects in advanced and metastatic settings (7), and there is accumulating evidence regarding the use of immunotherapy in treating the more indolent patient groups. These advancements have triggered an increasing interest in the use of immunotherapeutic agents for NMIBC, and especially BCG unresponsive NMIBC. Preliminary results of studies investigating the use of immunotherapeutic agents for the treatment of BCG unresponsive NMIBC have been recently released, which demonstrate the promise of immunotherapy in treating BCG unresponsive NMIBC (8). Numerous studies are being presently conducted in this field, and the results of these studies are awaited. The results of these studies can alter the current treatment strategies for BCG unresponsive NMIBC, and NMIBC in general, and clinicians need to consider such immunotherapeutic strategies for the treatment of BCG unresponsive NMIBC.

This review aimed to discuss the cutting-edge knowledge on the role of immunotherapy in BCG unresponsive NMIBC. The definition of BCG unresponsive NMIBC and the current treatment strategies for the management of BCG unresponsive NMIBC are summarized herein. The background for the use of immunotherapy for BCG unresponsive NMIBC is briefly discussed. We have additionally summarized the ongoing studies that are investigating the efficacy of immunotherapeutic agents for the treatment of BCG unresponsive NMIBC and BCG naive NMIBC.

\section{Definition of BCG unresponsive NMIBC}

Patients who fail during or after BCG immunotherapy require alternative treatment strategies. The definition of BCG failure is vital for assessing disease prognosis. BCG failure can be categorized into four subgroups according to the timing and nature of recurrence, and includes the "BCG intolerance", "BCG refractory", "BCG relapse", and "BCG unresponsive" subgroups (9). The term "BCG intolerant" is used when a patient is unable to receive the optimal BCG therapy due to adverse effects, and the BCG therapy has to be ceased (6). Although there can be differences in the dosage and duration, optimal BCG therapy must comprise induction therapy followed by maintenance therapy in patients with high risk (6). The "BCG refractory" subgroup comprises patients with persistent high-grade cancer following 6 months of induction therapy, or patients with cancers that have progressed 3 months after the commencement of induction therapy (10). "BCG relapse" indicates the recurrence of cancer after achieving a disease-free state after 6 months of BCG therapy. The "BCG refractory" subgroup generally comprises patients with poorer prognosis than the "BCG relapse" subgroup; however, "BCG relapse" within 6 months of last BCG exposure shows similar poor prognosis as the "BCG refractory" subgroup (9). In a previous prospective study, the survival of patients belonging to the BCG refractory subgroup was worse in comparison to that of the patients in the BCG relapse subgroup (11). In other words, as there are diverse definitions of BCG failure, the prognosis differs according to the definition of BCG failure (12).

For this reason, the International Bladder Cancer Group (IBCG) and the Genitourinary American Society of Clinical Oncology Group announced a novel, combined definition for the most aggressive subgroup of BCG failure, namely, the "BCG unresponsive" subgroup. According to their definition, BCG unresponsiveness is defined by the presence of persistent high-grade disease, or disease recurrence within 6 months of receiving at least two courses of intravesical instillation of BCG, after at least 5 of 6 induction doses along with at least 2 of 3 maintenance doses, or T1 high-grade disease at first evaluation following the induction of BCG alone after at least 5 of 6 induction doses. The BCG unresponsive subgroup of NMIBC specifically comprises patients with high risk of progression, for whom additional BCG therapy is unfeasible (1). This novel category is important for identifying what truly defines the failure of BCG therapy, which requires optimal treatment strategies following the discontinuation of BCG immunotherapy.

\section{Current treatment options for BCG unresponsive NMIBC}

Non-surgical treatment options for the treatment of patients who have failed on BCG therapy are limited, and radical cystectomy is therefore the most preferred option (5). Early radical cystectomy following BCG failure has been demonstrated to have better survival gains than delayed cystectomy $(13,14)$. The risk of progression of NMIBC to muscle-invasive bladder cancer (MIBC) is another evidence that supports the benefits of radical cystectomy in patients 
who have failed on BCG therapy (15). However, although early radical cystectomy has been shown to have survival benefits for patients who have failed on BCG therapy, the risks and benefits of cystectomy need to be carefully considered and balanced, owing to the high treatmentrelated morbidity and mortality $(16,17)$.

Although repeated BCG instillation is not recommended, it is an affordable option for a highly selected population. In persistent tumors, secondary BCG inductions have an approximately $50 \%$ response rate following a single induction of BCG (18). However, more than three induction therapies are not recommended owing to a low response rate of $20 \%$, and higher BCG-related toxicity $(18,19)$. Chemo-radiation therapy is a potential alternative for early radical cystectomy with a complete response rate of $88 \%$ and a 5 -year cancer specific survival rate of $84 \%$ (20). However, the toxicity of chemotherapeutic agents needs to be carefully considered because radiotherapy alone has no oncologic benefits for the treatment of NMIBC (21). Additionally, the role of chemoradiation therapy for patients with extensive carcinoma in situ remain to be determined (22).

Intravesical chemotherapy is another option for the treatment of patients who have failed on BCG and are unable to tolerate radical cystectomy. Valrubicin is the only approved chemotherapeutic agent for the treatment of patients who have failed on BCG therapy and have extensive carcinoma in situ. However, disease free rates of 6 months and 12 months are less than 20\% (9,23). Recently, intravesical gemcitabine and docetaxel chemotherapy has been demonstrated to be effective in patients who have failed on BCG therapy (24). An early phase III trial demonstrated that intravesical gemcitabine has better efficacy than intravesical mitomycin C in a BCG refractory setting (25). A previous study reported that the recurrence free rate following intravesical gemcitabine therapy is superior to that following secondary BCG induction (26). The oncological outcome of intravesical docetaxel therapy is promising with a complete response rate of $56 \%$ within 6 weeks of induction therapy in phase I trial (27). Additionally, the follow-up results demonstrated that the long-term outcomes of intravesical docetaxel therapy are good, with $69 \%$ of patients being able to avoid cystectomy at 2 -years (28). The combination of intravesical chemotherapy with other therapeutic strategies is gaining attention in the recent years. The most frequent combination is the sequential administration of gemcitabine/mitomycin C $(29,30)$ or gemcitabine/docetaxel $(31,32)$. Both the combination therapies show promising results in settings of BCG failure, however, as the oncological outcomes of intravesical chemotherapy are inferior to those of radical cystectomy, more reliable treatment strategies are necessary.

\section{Background for immunotherapy in BCG unresponsive NMIBC}

After the first intravesical BCG clinical trial by Morales and coworkers in 1976 (33), Lamm and coworkers performed a prospective randomized clinical trial to confirm the effects of BCG instillation in bladder cancer (34). Based on these results, BCG instillation has been regarded as the standard treatment for superficial bladder cancer (6), and the mechanism of action of BCG via stimulation of the immune system is well established (35). However, around $30 \%$ of patients with NMIBC do not respond to BCG and progress towards MIBC (36). These results triggered an increasing interest in novel agents for the treatment of unresponsive NMIBC, and the instillation of certain chemotherapeutic agents were subsequently studied. However, it was observed that the instillation of any single chemotherapeutic agent is unable to surpass the efficacy of BCG instillation (4). These historical and clinical studies supported the use of other immunotherapeutic agents for the treatment of systemic bladder cancer.

The identification of novel immunotherapeutic targets is important for the development of immunotherapeutic drugs. The genes that are involved in T-cell regulation were discovered in the 1980s using molecular biological techniques (37), and tumor inhibition using anti-cytotoxic T-lymphocyte antigen 4 (CTLA4) antibodies was reported in 1996 (38). Subsequently, several other immune checkpoints were sequentially identified. Currently, the primary immune checkpoints are anti-CTLA4, antiprogrammed cell death 1 (PD-1), and anti-programmed cell death ligand 1 (PDL-1) (39). The discovery of these immune checkpoints, which regulate the immune response, resulted in the development of treatment strategies that can be positively exploited for influencing T-cell activity and generating clinically relevant antitumor activities. These discoveries triggered the approval of immune check point inhibitors as a novel class of immunotherapy, that were first approved by the U.S. Food and Drug Administration (FDA) in 2011.

These immune check point inhibitors were investigated for treating several types of cancer and were reported to be effective for treating tumors with high mutation rates. A study by The Cancer Genome Atlas (TCGA) revealed that 
bladder cancer has a high mutation rate, with a mean of 7.7 per $\mathrm{Mb}$ within the coding regions (40). As aforementioned, bladder cancer has been reported to have a high mutational load, following adenocarcinoma of the lung, squamous cell carcinoma of the lung, and melanoma, among others. These genomic and epigenomic alterations in bladder cancer result in the profound formation of neoantigens, which are regarded as foreign proteins by the immune system. Thus, a high mutation rate and the increased formation of neoantigens in bladder cancer are associated with antitumor immune reactions, which enhance the effects of immune checkpoint inhibitors. Apart from these historical and clinical studies, recent advances in biology also support the use of other immunotherapeutic agents for the treatment of bladder cancer, and immune check point inhibitors have been investigated for the treatment of patients with bladder cancer.

Initially, the efficacy of immunotherapy in treating previous systemically-treated MIBC was investigated, which yielded promising results. Based on these results, atezolizumab, a well-known anti-PDL-1 agent, was approved as a second-line therapy for MIBC in 2016 (41). Subsequently, other immune checkpoint inhibitors, including nivolumab, durvalumab, avelumab, and pembrolizumab, have been sequentially approved as secondline therapy for bladder cancer (42-45). Additionally, atezolizumab and pembrolizumab were approved as firstline therapy for cisplatin-ineligible bladder cancer $(46,47)$. Following approval, the targets for immunotherapeutic agents were rapidly targeted for the treatment of NMIBC. The first targets for immunotherapy against NMIBC included targets for BCG unresponsive NMIBC as there were no satisfactory treatment options for BCG unresponsive NMIBC, and can advance the development of therapeutic targets for immunotherapy.

\section{Immunotherapy for BCG unresponsive NMIBC: ongoing trials}

The recent preliminary results of pembrolizumab, in addition to the recently published results of the study on high-risk BCG unresponsive patients, who declined to undergo or were ineligible for cystectomy, indicated that the treatment options investigated in the studies were superior to the currently available treatment strategies. The study reported that pembrolizumab induced a complete response in nearly $40 \%$ of patients with BCG unresponsive NMIBC. Grade 3-4 treatment-related adverse events were observed in $12.7 \%$ of the study population, although treatmentrelated adverse events of any grade were observed in $64.7 \%$ of the study population. In other words, it is thought that immunotherapy is effective for treating BCG unresponsive bladder cancer, and could offer oncologically superior and safe treatment options for the treatment of these patients in the near future, although the results from phase 3 randomized clinical trials are awaited.

Numerous studies are presently evaluating the effects of various immunotherapeutic agents for the treatment of BCG unresponsive NMIBC, which are summarized in Table 1. Two ongoing phase 3 randomized studies are evaluating pembrolizumab or nivolumab for the treatment of BCG unresponsive NMIBC, and the results of these studies are awaited. The results of these studies are expected to be published within the next few years, and these results are expected to massively alter the treatment pattern for BCG unresponsive NMIBC. Several simultaneous phase 2 studies on numerous immunotherapeutic agents combined with or without various types of drugs are being currently performed, and the results of these studies are expected to be published within 5 years. Other types of immunotherapeutic agents are also being currently investigated. For instance, BCG in combination with ALT803, a pharmacological grade IL-15/IL $15 \mathrm{R} \alpha$ complex fused to an IgG1 Fc, in which IL-15 is mutated to further increase the biological activity and agonism of the IL-2 and $15 \beta \gamma$ receptors, is also being evaluated in clinical trials, and the development of other types of immunotherapeutic agents are awaited.

Other immunotherapy using vaccine and gene therapy is currently evaluated for BCG unresponsive NMIBC. Vaccine is theoretically regarded as an attractive option for BCG unresponsive NMIBC because lifelong defense mechanism for cancer could be obtained using vaccine treatment. However, majority ongoing clinical trials using vaccine, such as Ty $21 \mathrm{a}$, a live attenuated bacterial vaccine that protects against typhoid, and RUTIVAC-1 is currently focused on BCG naive bladder cancer (48). For patients with NMIBC exposed to prior BCG, the results of PANVAC, a recombinant virus vector vaccine containing gene for human carcinoembryonic antigen, mucin-1 and costimulatory molecules, combined with BCG, are awaited (48). Gene therapy, another novel stream treatment method for BCG unresponsive NMIBC, is treatment methods using nucleic acid delivered into a host's cell, which was usually recruited for treating genetic disorders. Among gene therapy, immunogene therapy is targeting the 
Table 1 Ongoing studies using immunotherapeutic agents for BCG-unresponsive

\begin{tabular}{|c|c|c|c|c|c|c|}
\hline Identifier & Agents & Phase & Estimated number & Intervention model & Masking & Estimated completion \\
\hline NCT04149574 & $\mathrm{BCG} \pm$ nivolumab & 3 & 700 & Parallel (randomized) & Double blind & August 16, 2030 \\
\hline NCT03022825 & ALT-803 + BCG & 2 & 160 & Single group & Open label & September 2020 \\
\hline NCT02844816 & Atezolizumab & 2 & 202 & Single group & Open label & April 1, 2021 \\
\hline NCT03759496 & Durvalumab & 2 & 39 & Single group & Open label & December 31, 2021 \\
\hline NCT04164082 & Pembrolizumab + gemcitabine & 2 & 72 & Single group & Open label & March 31, 2023 \\
\hline NCT03519256 & Nivolumab \pm BMS-986205, BCG & 2 & 436 & Parallel (randomized) & Open label & June 3, 2023 \\
\hline NCT02625961 & Pembrolizumab & 2 & 260 & Single group & Open label & July 30, 2023 \\
\hline NCT04106115 & Durvalumab + S-488210/S488211 & $1 \mathrm{~b} / 2$ & 64 & Single group & Open label & February 2027 \\
\hline NCT02808143 & Pembrolizumab + BCG & 1 & 27 & Single group & Open label & February 2020 \\
\hline NCT03258593 & Durvalumab + vicinum & 1 & 40 & Sequential assignment & Open label & December 30, 2022 \\
\hline NCT03892642 & Avelumab + BCG & $1 b$ & 27 & Single group & Open label & October 2025 \\
\hline
\end{tabular}

BCG, Bacillus Calmette-Guérin.

immune system and, in this regards, immunogene therapy could be categorized into immunotherapy. Recently, an interim results of phase 2 study for CG0070, a replicationcompetent oncolytic adenovirus, showed overall 6 months complete response rate of $47 \%$ in BCG unresponsive NMIBC patients with acceptable toxicity (49) Another gene therapeutic agent using adenovirus showed $35 \%$ high grade recurrent-free rate at 12 months without tolerable side effects in patients with BCG unresponsive NMIBC (50). Currently, phase 3 study using INSTILADRIN is on the way and the study expected to complete in 2022 .

\section{Immunotherapy for BCG unresponsive NMIBC: future directions}

Immunotherapy could be the mainstream treatment option or one of the standard treatment options not only for MIBC, but also for BCG unresponsive NMIBC, if the results of the current ongoing trials, which are expected in a few years, are promising. BCG naive NMIBC could serve as the other target for immunotherapy in the near future, although some biomarkers are necessary for selecting the appropriate treatment targets for optimizing the individualized treatment options for patients with bladder cancer. In addition, due to the world-wide BCG shortage, the novel treatment methods with reliable effects for BCG naive NMIBC is desperately awaited.

Some studies have aimed to evaluate the effects of immunotherapeutic agents on BCG naive NMIBC (Table 2). However, with the exception of one phase 3 study on durvalumab, the other studies on immune checkpoint inhibitors are small phase 1 studies and larger studies with reliable study designs are necessary for validating the effects of immunotherapy on BCG naive NMIBC, on the basis of these phase 1 studies. Additionally, other types of immunotherapeutic agents are also being tested for the treatment of BCG naive NMIBC, and the results of a phase $1 \mathrm{~b} / 2 \mathrm{~b}$ ALT-803 study on 596 patients are awaited. In addition, gene therapy could be another novel stream treatment method for BCG unresponsive NMIBC, as mentioned above, although optimal selection strategies for each patient are remained to be developed. These studies could mark the beginning of massive alterations and a number of studies on immunotherapeutic agents are expected to be performed within a few years the treatment of BCG naive NMIBC, and it is necessary for clinicians to be concerned with these aspects.

In near future, vaccine might be an effective preventing 
Table 2 Ongoing studies using immunotherapeutic agents for BCG-naive NMIBC

\begin{tabular}{lllllll}
\hline Identifier & Agents & Phase & Estimated number & Intervention model & Masking & Estimated completion \\
\hline NCT03528694 & BCG \pm durvalumab & 3 & 975 & Parallel (randomized) & Open label & November 25, 2024 \\
NCT02138734 & BCG \pm ALT-803 & 1b/2b & 596 & Parallel (randomized) & Open label & October 2021 \\
NCT04134000 & BCG + atezolizumab & 1 & 40 & Single group & Open label & November, 2022 \\
NCT02324582 & BCG + pembrolizumab & 1 & 15 & Single group & Open label & December 30, 2022 \\
NCT03421236 & Ty21a & 1 & 25 & Single group & Open label & March 1, 2021 \\
NCT03191578 & RUTIVAC-1 & 1 & 40 & Parallel (randomized) & Triple blind & March, 2023 \\
\hline
\end{tabular}

BCG, Bacillus Calmette-Guérin; NMIBC, non-muscle invasive bladder cancer.

method for bladder cancer, in addition to treatment methods for BCG naive NMIBC because of the lifelong prevention of bladder cancer as mentioned above. Currently, two ongoing phase 1 clinical trials using vaccine, including Ty $21 \mathrm{a}$, and RUTIVAC-1 is on the way and the results are awaited. If these studies showed promising results, not only treatment landscape for NMIBC, but also preventing strategies for bladder cancer expected to change significantly.

\section{Conclusions}

At present, the treatment of $B C G$ unresponsive NMIBC is challenging, and novel treatment options for BCG unresponsive NMIBC awaited. A number of immunotherapeutic agents have been recently developed, which showed satisfactory oncological outcomes for the treatment of MIBC and are rapidly progressing towards the treatment of BCG unresponsive NMIBC. A large number of clinical trials are ongoing and the preliminary results of some of these trials have been promising, which might alter the treatment strategies for BCG unresponsive NMIBC in a few years. Additionally, the treatment landscape for BCG unresponsive NMIBC, and also for NMIBC in general, could undergo slight alterations in a few years. In addition, due to the worldwide shortage of the BCG the development of novel agents for not only for BCG unresponsive NMIBC, but also for BCG naive NMIBC. In this regard, clinicians need to continue studying clinical trials on the use of immunotherapy in treating BCG unresponsive NMIBC.

\section{Acknowledgments}

Funding: None.

\section{Footnote}

Provenance and Peer Review: This article was commissioned by the Guest Editors (Dr. Ja Hyeon Ku, Dr. Hyeong Dong Yuk, and Dr. Hyung Suk Kim) for the series "Urothelial Carcinoma" published in Translational Cancer Research. The article was sent for external peer review organized by the Guest Editors and the editorial office.

Conflicts of Interest: Both authors have completed the ICMJE uniform disclosure form (available at http:// dx.doi.org/10.21037/tcr-20-758). The series "Urothelial Carcinoma" was commissioned by the editorial office without any funding or sponsorship. The authors have no conflicts of interest to declare.

Ethical Statement: The authors are accountable for all aspects of the work in ensuring that questions related to the accuracy or integrity of any part of the work are appropriately investigated and resolved.

Open Access Statement: This is an Open Access article distributed in accordance with the Creative Commons Attribution-NonCommercial-NoDerivs 4.0 International License (CC BY-NC-ND 4.0), which permits the noncommercial replication and distribution of the article with the strict proviso that no changes or edits are made and the original work is properly cited (including links to both the formal publication through the relevant DOI and the license). See: https://creativecommons.org/licenses/by-nc-nd/4.0/.

\section{References}

1. Kamat AM, Hahn NM, Efstathiou JA, et al. Bladder cancer. Lancet 2016;388:2796-810. 
2. van Rhijn BW, Burger M, Lota $Y$, et al. Recurrence and progression of disease in non-muscle-invasive bladder cancer: from epidemiology to treatment strategy. Eur Urol 2009;56:430-42.

3. Sylvester RJ, van der Meijden AP, Oosterlinck W, et al. Predicting recurrence and progression in individual patients with stage Ta T1 bladder cancer using EORTC risk tables: a combined analysis of 2596 patients from seven EORTC trials. Eur Urol 2006;49:466-5.

4. Chou R, Selph S, Buckley DI, et al. Intravesical Therapy for the Treatment of Nonmuscle Invasive Bladder Cancer: A Systematic Review and Meta-Analysis. J Urol 2017;197:1189-99.

5. Woldu SL, Bagrodia A, Lotan Y, et al. Guideline of guidelines: non-muscle-invasive bladder cancer. BJU Int 2017;119:371-80.

6. Babjuk M, Burger M, Comperat EM, et al. European Association of Urology Guidelines on Non-muscleinvasive Bladder Cancer (TaT1 and Carcinoma In Situ) 2019 Update. Eur Urol 2019;76:639-57.

7. Stenehjem DD, Tran D, Nkrumah MA, et al. PD1/PDL1 inhibitors for the treatment of advanced urothelial bladder cancer. Onco Targets Ther 2018;11:5973-89.

8. Kulkarini GS, de Wit R, Balar AV, et al. MP43-01 Phase 2 KEYNOTE-057 STUDY: pembrolizumab for patients with high-risk non-muscle invasive bladder cancer unresponsive to bacillus Calmette-Guerin. J Urol 2019;201:e616.

9. Kamat AM, Colombel M, Sundi D, et al. BCGunresponsive non-muscle-invasive bladder cancer: recommendations from the IBCG. Nat Rev Urol 2017;14:244-55.

10. Jarow JP, Lerner SP, Kluetz PG, et al. Clinical trial design for the development of new therapies for nonmuscleinvasive bladder cancer: report of a Food and Drug Administration and American Urological Association public workshop. Urology 2014;83:262-4.

11. Herr HW, Milan TN, Dalbagni G. BCG-refractory vs. BCG-relapsing non-muscle-invasive bladder cancer: a prospective cohort outcomes study. Urol Oncol 2015;33:108.e1-4.

12. Kamat AM, Sylvester RJ, Böhle A, et al. Definitions, End Points, and Clinical Trial Designs for Non-MuscleInvasive Bladder Cancer: Recommendations From the International Bladder Cancer Group. J Clin Oncol 2016;34:1935-44.

13. Herr HW, Sogani PC. Does early cystectomy improve the survival of patients with high risk superficial bladder tumors? J Urol 2001;166:1296-9.

14. Huguet J, Crego M, Sabaté S, et al. Cystectomy in patients with high risk superficial bladder tumors who fail intravesical BCG therapy: pre-cystectomy prostate involvement as a prognostic factor. Eur Urol 2005;48:539; discussion 59.

15. Tilki D, Reich Om Svatek RS, et al. Characteristics and outcomes of patients with clinical carcinoma in situ only treated with radical cystectomy: an international study of 243 patients. J Urol 2010;183:1757-63.

16. Leow JJ, Reese SW, Jiang W, et al. Propensitymatched comparison of morbidity and costs of open and robot-assisted radical cystectomies: a contemporary population-based analysis in the United States. Eur Urol 2014;66:569-76.

17. Stimson CJ, Chang SS, Barocas DA, et al. Early and late perioperative outcomes following radical cystectomy: 90-day readmissions, morbidity and mortality in a contemporary series. J Urol 2010;184:1296-300.

18. Dalbagni G, Herr HW. Current use and questions concerning intravesical bladder cancer group for superficial bladder cancer. Urol Clin North Am 2000;27:137-46.

19. Catalona WJ, Hudson MA, Gillen DP, et al. Risks and benefits of repeated courses of intravesical bacillus Calmette-Guerin therapy for superficial bladder cancer. J Urol 1987;137:220-4.

20. Weiss C, Wolze C, Engehausen DG, et al. Radiochemotherapy after transurethral resection for high-risk $\mathrm{T} 1$ bladder cancer: an alternative to intravesical therapy or early cystectomy? J Clin Oncol 2006;24:2318-24.

21. Harland SJ, Kynaston H, Grigor K, et al. A randomized trial of radical radiotherapy for the management of pT1G3 NXM0 transitional cell carcinoma of the bladder. J Urol 2007;178:807-13; discussion 813.

22. Shipley WU, Rose MA, Perrone TL, et al. Full-dose irradiation for patients with invasive bladder carcinoma: clinical and histological factors prognostic of improved survival. J Urol 1985;134:679-83.

23. Dinney CP, Greenberg RE, Steinberg GD, et al. Intravesical valrubicin in patients with bladder carcinoma in situ and contraindication to or failure after bacillus Calmette-Guérin. Urol Oncol 2013;31:1635-42.

24. Peyton CC, Chipollini J, Azizi M, et al. Updates on the use of intravesical therapies for non-muscle invasive bladder cancer: how, when and what. World J Urol 2019;37:2017-29.

25. Addeo R, Caraglia M, Bellini S, et al. Randomized phase 
III trial on gemcitabine versus mytomicin in recurrent superficial bladder cancer: evaluation of efficacy and tolerance. J Clin Oncol 2010;28:543-8.

26. Di Lorenzo G, Perdonà S, Damiano R, et al. Gemcitabine versus bacille Calmette-Guérin after initial bacille Calmette-Guérin failure in non-muscle-invasive bladder cancer: a multicenter prospective randomized trial. Cancer 2010;116:1893-900.

27. Laudano MA, Barlow LJ, Murphy AM, et al. Long-term clinical outcomes of a phase I trial of intravesical docetaxel in the management of non-muscle-invasive bladder cancer refractory to standard intravesical therapy. Urology 2010;75:134-7.

28. Barlow LJ, McKiernan JM, Benson MC, et al. Long-term survival outcomes with intravesical docetaxel for recurrent nonmuscle invasive bladder cancer after previous bacillus Calmette-Guérin therapy. J Urol 2013;189:834-9.

29. Lightfoot AJ, Breyer BN, Rosevear HM, et al. Multiinstitutional analysis of sequential intravesical gemcitabine and mitomycin C chemotherapy for non-muscle invasive bladder cancer. Urol Oncol 2014;32:35.e15-9.

30. Cockerill PA, Knoedler JJ, Frank I, et al. Intravesical gemcitabine in combination with mitomycin $\mathrm{C}$ as salvage treatment in recurrent non-muscle-invasive bladder cancer. BJU Int 2016;117:456-62.

31. Velaer KN, Steinberg RL, Thomas LJ, et al. Experience with Sequential Intravesical Gemcitabine and Docetaxel as Salvage Therapy for Non-Muscle Invasive Bladder Cancer. Curr Urol Rep 2016;17:38.

32. Milbar N, Kates M, Chappidi MR, et al. Oncological Outcomes of Sequential Intravesical Gemcitabine and Docetaxel in Patients with Non-Muscle Invasive Bladder Cancer. Bladder Cancer 2017;3:293-303.

33. Morales A, Eidinger D, Bruce AW. Intracavitary Bacillus Calmette-Guerin in the Treatment of Superficial Bladder Tumors. J Urol 2017;197:S142-5.

34. Lamm DL. Bacillus Calmette-Guerin immunotherapy for bladder cancer. J Urol 1985;134:40-7.

35. Pettenati C, Ingersoll MA. Mechanisms of BCG immunotherapy and its outlook for bladder cancer. Nat Rev Urol 2018;15:615-25.

36. Gandhi NM, Morales A, Lamm DL. Bacillus CalmetteGuérin immunotherapy for genitourinary cancer. BJU Int 2013;112:288-97.

37. Reinherz EL, Schlossman SF. The differentiation and function of human T lymphocytes. Cell 1980;19:821-7.

38. Leach DR, Krummel MF, Allison JP Enhancement of antitumor immunity by CTLA-4 blockade. Science
1996;271:1734-6.

39. Postow MA, Callahan MK, Wolchok JD. Immune Checkpoint Blockade in Cancer Therapy. J Clin Oncol 2015;33:1974-82.

40. Cancer Genome Atlas Research Network. Comprehensive molecular characterization of urothelial bladder carcinoma. Nature 2014;507:315-22.

41. Rosenberg JE, Hoffman-Censits J, Powles T, et al. Atezolizumab in patients with locally advanced and metastatic urothelial carcinoma who have progressed following treatment with platinum-based chemotherapy: a single-arm, multicentre, phase 2 trial. Lancet 2016;387:1909-20.

42. Bellmunt J, de Wit R, Vaughn DJ, et al. Pembrolizumab as Second-Line Therapy for Advanced Urothelial Carcinoma. N Engl J Med 2017;376:1015-26.

43. Sharma P, Retz M, Siefker-Radtke A, et al. Nivolumab in metastatic urothelial carcinoma after platinum therapy (CheckMate 275): a multicentre, single-arm, phase 2 trial. Lancet Oncol 2017;18:312-22.

44. Powles T, O'Donnell PH, Massard C, et al. Efficacy and safety of durvalumab in locally advanced or metastatic urothelial carcinoma: updated results from a phase 1/2 open-label study. JAMA oncology JAMA Oncol 2017;3:e172411.

45. Apolo AB, Infante JR, Balmanoukian A, et al. Avelumab, an Anti-Programmed Death-Ligand 1 Antibody, In Patients With Refractory Metastatic Urothelial Carcinoma: Results From a Multicenter, Phase Ib Study. J Clin Oncol 2017;35:2117-24.

46. Balar AV, Castellano D, O'Donnell PH, et al. First-line pembrolizumab in cisplatin-ineligible patients with locally advanced and unresectable or metastatic urothelial cancer (KEYNOTE-052): a multicentre, single-arm, phase 2 study. Lancet Oncol 2017;18:1483-92.

47. Balar AV, Galsky MD, Rosenberg JE, et al. Atezolizumab as first-line treatment in cisplatin-ineligible patients with locally advanced and metastatic urothelial carcinoma: a single-arm, multicentre, phase 2 trial. Lancet 2017;389:67-76.

48. Packiam VT, Werntz RP, Steinberg GD. Current Clinical Trials in Non-muscle-Invasive Bladder Cancer: Heightened Need in an Era of Chronic BCG Shortage. Curr Urol Rep 2019;20:84.

49. Packiam VT, Lamm DL, Barocas DA, et al. An open label, single-arm, phase II multicenter study of the safety and efficacy of CG0070 oncolytic vector regimen in patients with BCG-unresponsive non-muscle-invasive bladder 
cancer: Interim results. Urol Oncol 2018;36:440-7.

50. Shore ND, Boorjian SA, Canter DJ, et al. Intravesical rAd-IFN $\alpha /$ Syn 3 for Patients With High-Grade, Bacillus
Calmette-Guerin-Refractory or Relapsed Non-MuscleInvasive Bladder Cancer: A Phase II Randomized Study. J Clin Oncol 2017;35:3410-6.

Cite this article as: Suh J, Yoo S. Role of immunotherapy in Bacillus Calmette-Guérin unresponsive: non-muscle invasive bladder cancer. Transl Cancer Res 2020;9(10):6537-6545. doi: $10.21037 /$ tcr- $20-758$ 